\title{
La violencia intrafamiliar en el desempeño laboral de las mujeres en el sector textil-confección ${ }^{*}$
}

\section{Domestic violence in the labor performance of women in the textile-confection industry}

Luis Fernando Quintero Arango** ORCID: 0000-0002-6268-065X

Carlos Eduardo Álvarez Agudelo ORCID: 0000-0002-1426-0343

Diana Marcela Ibagon Parra ORCID: 0000-0003-4196-970X Universidad Católica Luis Amigó, Colombia

Recibido: 18 de noviembre de 2018 Revisado: 6 de febrero de 2019 Aceptado: 4 de abril de 2019

\section{Resumen}

El objetivo principal de la investigación fue conocer los factores que se derivan de la violencia intrafamiliar y la incidencia en la productividad laboral en empresas del sector textil confección. Inicialmente, se realizó una revisión de la literatura, para luego desarrollar el proyecto investigativo bajo el paradigma interpretativo, haciendo énfasis, en identificar cómo el fenómeno de la violencia intrafamiliar impacta el desempeño laboral de las trabajadoras de este tipo de empresas. La metodología fue cualitativa, con un diseño transversal, y se utilizó la técnica de recolección de información por medio de un grupo focal y una entrevista semiestructurada con preguntas orientadoras. En los resultados se pudo constatar que la violencia intrafamiliar es un factor que incide en el bajo nivel de desempeño y productividad de las trabajadoras; donde el desempeño laboral se ve afectado por esta situación impactando la baja calidad en el trabajo. Así mismo, se estableció que las

Artículo de investigación. Cómo citar: Quintero, A. L. F., Álvarez, A. C. E., \& Ibagon, P. D. M. (2019). La violencia intrafamiliar en el desempeño laboral de las mujeres en el sector textil-confección. Diversitas: Perspectivas en Psicología, 15(2), 271-284. DOI: 10.15332/22563067.5402 Este trabajo es producto del proyecto de investigación "Violencia de género en el ámbito intrafamiliar y su incidencia en la productividad laboral en 24 mujeres de las ciudades de Bogotá, Medellín y Manizales. Análisis comparativo". Financiado por la Universidad Católica Luis Amigó. Correspondencia: Luis Fernando Quintero Arango, Docente, Investigador. Universidad Católica Luis Amigó. Correo electrónico: luis.quinterora@ amigo.edu.co. Dirección postal: Facultad de Ciencias Económicas, Administrativas y Contables. Programa de Administración de Empresas. Bloque 4, P. 2 aula 203. Universidad Católica Luis Amigó. Transversal 51A n. ${ }^{\circ 67 B-90, ~ M e d e l l i ́ n, ~ C o l o m b i a . ~}$ 
organizaciones no cuentan con una ruta de atención que ofrezca apoyo a las mujeres víctimas de la violencia intrafamiliar.

Palabras clave: productividad; violencia intrafamiliar; mujeres; sector textil, confección.

\section{Abstract}

The main objective of the research was to know the factors that are derived from intrafamily violence and the incidence of labor productivity in companies in the textile clothing industry. Initially, a review of the literature was made, to then develop the research project under the interpretive paradigm, emphasizing, in identifying how the phenomenon of intrafamily violence impacts the work performance of workers of this type of companies. The methodology was a qualitative approach, a cross-sectional design, and the technique of gathering information was used through a focus group and a semi-structured interview with guiding questions. In the results obtained, it was found that intrafamily violence is a factor that affects the low level of performance and productivity in workers; where the work performance is affected by this situation having impact on the low quality at work. Likewise, it was established that organizations do not have a care route that offers support to women victims of domestic violence.

Keywords: Productivity, domestic violence, women, textile manufacturing.

\section{Introducción}

Al abordar el tema de la productividad y la relación con la violencia intrafamiliar, se analizó que las mujeres que laboran en el sector textil, confección y que han sufrido de forma directa la violencia que proviene del comportamiento familiar, podría incidir en su rendimiento laboral (González, 2018). Esto se puede evidenciar por medio de indicadores de gestión cómo son; el rendimiento productivo, horarios de trabajo, hora de trabajo - máquina e incapacidades emitidas por el sector formal de salud y otras que no están legalmente aprobadas por las instituciones prestadoras de salud. Así mismo, derivado de esa violencia, aparecen efectos desde el aspecto psicológico como son: bajo estado de ánimo, conductas agresivas en el trabajo, una sensación de temor por el que dirán de la situación vivida y la preocupación por un posible despido por la realidad que se presenta en su entorno familiar.

El sector textil-confección genera empleo formal, especialmente para las mujeres. Según Campos (2014) las mujeres aportan alrededor del $35 \%$ de la mano de obra para este sector en Colombia. Pero, factores que se presentan derivados de los efectos de la violencia intrafamiliar afectan no solo, la baja productividad, sino las pérdidas económicas ocasionadas por las trabajadoras cuando se vinculan aspectos como desmotivación, preocupación, estrés y somatización de enfermedades desencadenantes de este tipo de vivencias. Esto incide en la calidad de los productos terminados, ya que estos pueden resultar defectuosos y serán considerados por la empresa sinónimos de mala calidad, incrementando los costos en la producción (Castillo, 2011).

Así mismo, las empresas que tienen dificultades en la productividad por falta de situaciones están relacionados con problemas familiares impactan de forma negativa los resultados que la compañía espera y tiene contemplado dentro de sus presupuestos (Campos, 2014).

En el desempeño laboral se considera importante medir la productividad, para Cruelles (2012) la productividad se mide por el tiempo en que una persona invierte en realizar un producto, en donde, a mayor incremento de la producción en un menor tiempo se logra ser eficiente, impactando así la dis- 
minución de los costos de producción, convirtiendo su proceso competitivo y eficaz. Pero, una mujer que haya sufrido violencia intrafamiliar, reflejará su baja productividad por los efectos que se deriven de ese acto de violencia y en el descenso de los indicadores de producción según lo determina la empresa.

Por otro lado, Casique (2014) relaciona la violencia de género con los efectos laborales ya que existe una incidencia de doble vía entre los ingresos (salario) y la condición de ejercer su actividad laboral de forma continua, ya que se pueden presentar incapacidades médicas, que impactan el presupuesto financiero, derivado de los efectos de la violencia, que se puede manifestar de forma física, verbal, psicológica o sexual. Lo anterior, puede deteriorar la calidad de vida y las relaciones interpersonales con los compañeros de trabajo, tomando la decisión de renunciar y pudiendo afectar de manera directa su ingreso familiar.

Para el desarrollo de este artículo se partió del objetivo de la investigación que fue conocer los factores que se derivan de la violencia intrafamiliar y la incidencia en la productividad laboral, un análisis en empresas del sector textil-confección de Bogotá, Medellín y Manizales. Para el estudio del fenómeno de la violencia intrafamiliar se elaboró la clasificación de las siguientes categorías: tipología de la violencia, infractor de la violencia y estado emocional post violencia. Esto se realizó por medio de una entrevista semiestructurada con preguntas orientadoras, y tomando tres empresas del sector textil-confección, en las ciudades de Medellín, Bogotá y Manizales. La investigación tuvo un diseño transversal, es decir, en un solo momento entre el período comprendido entre febrero de 2016 hasta noviembre de 2016, las personas entrevistadas fueron operarias mayores de edad.

El artículo está estructurado de la siguiente forma. La primera parte explica la introducción, con los elementos principales propuestos desde la importancia del tema que se investigó y de la forma en que se realizó el diseño metodológico. El apartado número dos hace énfasis en el diseño metodológico, está investigación se realizó, con un enfoque cualitativo, una investigación de tipo de descriptivo, con un diseño transversal y de forma no experimental, utilizando la herramienta de grupo focal con una entrevista semiestructurada. La tercera parte, es el desarrollo del marco teórico, allí se aborda el tema del contexto familiar y de comportamiento frente al tema de la violencia intrafamiliar, y el tema relacionado con lo laboral y productivo. El cuarto apartado, trata de los hallazgos de la investigación, en donde se logró identificar los problemas que se presentan desde el hogar y la incidencia en la parte laboral y productiva, por último las conclusiones encontradas en el trabajo, se evidencia que la situación de la violencia intrafamiliar no es considerada como un factor que incide en la productividad de la empresa, donde los gerentes no tienen contemplado un plan de trabajo para tratar este tipo de situaciones, en las tres empresas donde se realizó la investigación no se encontraron rutas o programas para el manejo de la violencia intrafamiliar.

\section{Marco de referencia}

\section{Contexto familiar y de comportamiento}

La violencia intrafamiliar es una de las problemáticas más relevantes en Colombia, según el Instituto de Medicina Legal en el informe Forense (2015) entregan las siguientes cifras:

735 niñas y mujeres adolescentes fueron víctimas de violencia por parte de un familiar o cuidador y 6.269 fueron víctimas de violencia de pareja. En cuanto a las mujeres mayores de 60 años, 119 de ellas fueron agredidas físicamente y 1.482 fueron víctimas de violencia por parte de otros familiares. (p. 7)

Como se observa, según las cifras que nos da a conocer el Instituto de Medicina Legal (2015) la violencia intrafamiliar cobija a todos los grupos etarios dentro del contexto familiar, tanto del grupo nuclear primario, la relación conyugal o relación de pareja o con los cuidadores que representa esa red de apoyo. De este modo podemos ver como las cifras más elevadas están directamente relacionadas con el maltrato a niñas y mujeres adolescentes, las cuales han sido víctimas de violencia intrafamiliar 
por parte de un cuidador, un familiar y en la mayoría de casos han sido violentadas por una pareja, representado esto un total de 7004 niñas y mujeres adolescentes. Dentro de las principales formas de agresión se encuentran la violencia física y la violencia verbal las cuales tienen un alto impacto relacionado con el maltrato hacia mujeres mayores.

De otro lado, desde los imaginarios sociales, el establecimiento de los patrones de violencia de cualquier tipo, se han fundamentado dentro del concepto de "normalidad" en el ámbito familiar. Al respecto Castillo (2011) expresa que desde el núcleo familiar y los aspectos socioculturales violentos, inciden en la forma en que se desintegran las familias actuales, esto determinado por conductas agresivas, una comunicación no asertiva, y aspectos económicos y de ingresos que afectan al grupo familiar, especialmente, en aquellos donde la responsabilidad recae en una sola persona y especialmente que sea del género femenino.

Para entender el concepto de violencia intrafamiliar es necesario que se ahonde en el tema desde una dinámica vinculante, asociada a los patrones de respuesta que se establecen en el ámbito de familia. Al respecto Quiñones et al., (2011) explican que la violencia intrafamiliar es el abuso del poder y la confianza; la dinámica de la violencia es un ciclo que cuenta con tres fases:

Etapa 1 llamada fase acumulación de tenciones donde se produce una serie de incidentes donde cada vez se vuelven más hostiles que van desde agresiones hasta verbales hasta daños menores.

Etapa 2 se denomina episodio agudo de concreción; de la violencia es aquella en cual todas las tenciones que se acumulan estallan puede ir de empujones al homicidio o suicidio.

Etapa 3 denominado luna de miel es cuando se produce el arrepentimiento por parte del hombre a veces casi de forma inmediata el hombre llega a un periodo de seducción y la promesa que esto no volverá a ocurrir.

La mayor parte de la violencia intrafamiliar es violencia de género se traduce de violencia dirigida de un hombre hacia mujeres; los hijos deben respeto a los mayores y la mujer a su marido y él debe responder por toda la familia en definitiva los estereotipos de género en los que el valor masculino vale más que el femenino contribuyen a delimitar factores de riesgo (p. 245).

Teniendo en cuenta que la violencia afecta a todos los seres humanos, independientemente de su cultura, clase social, etnia, educación, género u orientación sexual, Hidalgo y Valdés (2014), consideran que la violencia es un detonante que tiene un desencadenamiento en el género femenino, que conlleva a que se produzca un daño psicológico, físico, sexual. Además, también se inician amenazas, limitaciones a la libertad e intimidaciones, convirtiendo esa violencia intrafamiliar en un elemento para desencadenar problemas mayores en la relación interna de cada familia.

Al abordar la problemática con las mujeres participantes, aunque el objetivo era determinar la incidencia de la violencia intrafamiliar en el desempeño laboral, se encontraron diversas formas de violencia en los diferentes roles que estas mujeres han sufrido a lo largo de su vida. Por ejemplo, algunas mujeres expresaban que no solo habían vivido la violencia en el ámbito conyugal, sino que también en contextos laborales; entendida esta violencia, como forma de discriminación. De igual forma, el principal agresor, aunque lo constituía el cónyuge no solamente este ha sido en su vida el único que ha generado agresión, los hermanos, el padre, padrastro, jefes, novios y en algunos casos familia extensa quien a partir de la crítica han ejercido el maltrato a estas mujeres (Casique, 2014).

Así mismo, en los datos recogidos y analizados se encontró que el concepto de violencia intrafamiliar se concreta y se define solo al momento de sufrir una agresión física, lo demás no es considerado como violencia. De allí, que se presente una homogeneidad en las respuestas de cada una de las mujeres participantes en las tres ciudades, asociadas a otros puntos en común como son: los modelos machistas que generan violencia y los patrones de respuesta de las mujeres frente a estos hechos. Al respecto, cuando además se trata de familias en las que acontecen situaciones de violencia doméstica, se 
incrementa el riesgo, se potencializa que la estructura familiar sufra un franco deterioro. Una muestra de esto lo es la violencia hacia las mujeres y niños, niñas y adolescentes (Pacheco \& Capano, 2014).

Estos modelos fueron encontrados en las tres ciudades, aunque no todas las mujeres indagadas han sufrido todas las formas de violencia (en su mayoría sí), se encontró que todas por lo menos una vez en su vida, tanto a nivel marital como en su familia nuclear, ha sufrido por lo menos algún tipo de violencia. Por otra parte, González (2018) indica que, aunque existe claridad del concepto y se percibe el conflicto en cuanto a hablar abiertamente sobre el tema. En este sentido se observó en las participantes auto-confrontación cuando comentaban en qué forma habían decidido resolver la problemática, las acciones que habían tomado y las que habían dejado de tomar, sobre todo se percibieron expresiones cargadas de vergüenza por parte de aquellas que aún continúan en la relación problemática.

En cuanto al tema de violencia intrafamiliar contra las mujeres en el país, Sánchez (2013) Director General del Centro Nacional de Memoria Histórica, dependencia que tiene vínculos internacionales con la Oficina en Colombia del Alto Comisionado de las Naciones Unidas para los Derechos Humanos, en el informe ¡basta ya! Colombia: Memorias de guerra y dignidad, expone:

Las mujeres y niñas, se siguen viendo involucradas y gravemente afectadas por la violencia física, verbal y psicológica. En el 2011 se presentaron en el país 89.807 casos de violencia intrafamiliar (VIF), siendo el 78,1\% mujeres y 57.761 como violencia de pareja. Estableciendo que el $37 \%$ de las mujeres Colombianas alguna vez han sufrido de una agresión física por parte de sus parejas "Encuesta Nacional de Demografía y Salud". Las dimensiones de la problemática en Bogotá son superiores, pues ocupa el primer lugar del país en denuncias sobre violencia contra niños, niñas y adolescentes. Presentándose en 20113.459 casos. A raíz del análisis de estos casos, se revelo en una encuesta realizada que el $6 \%$ de las mujeres encuestadas fueron abusadas sexualmente, específicamente por personas de su familia como (parientes, ex esposo, no- vio, padre, padrastro y hermano), únicamente el $21,5 \%$ se trató de un desconocido (p. 456).

La presente investigación estuvo enfocada en articular y reconocer los patrones de conducta que presentan las mujeres en el ámbito laboral y cómo estos inciden en su desempeño. Esto permite no solamente lograr el objetivo de la investigación, sino que también posibilita una exploración de formas de violencia y manejo de la problemática por estas mujeres, percibido este concepto desde el lente de las afectadas y su posición frente a los actos violentos y cómo estos han incidido en su vida en todas sus esferas, especialmente en la emocional, familiar, laboral y social.

Por otra parte, Álvarez y Gómez (2011) indican que el reconocimiento que se le dé a una mujer en el hogar por parte de su pareja, hijos, familia, es de vital importancia a la hora de desempeñarse en un cargo. Muchas de las mujeres que sufren de maltrato intrafamiliar están motivadas por la mejora de la calidad de vida de los integrantes de su hogar y de ella misma. Esto representa una motivación para emplearse y generar recursos económicos que estabilicen la calidad de vida de su grupo nuclear. Ahora bien, cuando existe violencia intrafamiliar bien sea física, verbal o en cualquiera de sus expresiones, esto incide de manera directa en la motivación que lleva a la mujer a desempeñarse en un ámbito laborar afectando en muchas ocasiones su rendimiento laboral y su tranquilidad ya que la familia repercute directamente en estabilidad emocional y no contar con herramientas que le permitan afrontar una problemática familiar donde se ve directamente afectada. Esto, teniendo en cuenta que la violencia de género, como forma de violencia presente en todo el mundo y producto de la desigualdad entre hombres y mujeres, genere violencia intrafamiliar y divorcio (García Figueroa, 2014).

Para Castro y Casique (2008) la violencia psicológica se refiere a las formas de agresión repetida que no inciden directamente en el cuerpo de la mujer, pero sí a nivel psicológico; al enviar un mensaje negativo que constantemente involucra aspectos tales como, amenazas, insultos, ofensas, palabras soeces; que llevan un mensaje violento y directo, 
Esta motivación es la que impulsa a laborar, de no ser así y haciendo énfasis que la violencia no es solo física si no también emocional, la repercusión se hace evidente en mujeres que desempeñan un cargo. Como se planteó anteriormente y de acuerdo a la presente investigación, al afrontar un problema familiar que en este caso sería sufrir violencia doméstica, influye en el desempeño, pues se hace muy difícil hacer una separación entre la vida laboral y familiar, teniendo en cuenta que la mujer que enfrenta maltrato es la misma que debe cumplir con un compromiso laboral y debe cumplir una meta o requerimiento por la entidad para la que labora. Se menciona esto porque se encontró en la investigación la atención insuficiente y el bajo apoyo a las mujeres víctimas de violencia intrafamiliar. Se encontró que las mujeres trabajadoras tenían serias dificultades para acceder a permisos y ausencias justificadas para realizar trámites de denuncias del abuso, de igual forma, las mujeres a quienes se les permitía la salida o faltar a su labor, deberían cumplir con la cuota de productividad en horarios extra laborales, lo que representa una revictimización de estas mujeres, este apartado se desarrollara con profundidad en el siguiente punto (González, 2018).

\section{Contexto laboral y productivo}

En esta apartado, se abordarán los factores laborales y productivos, y el rol de la mujer en el desempeño laboral colombiano, en el sector textil-confección.

En cuanto al contexto laboral y productivo se encuentran estudios en este ámbito en cuanto al rol de la mujer, Fuentes (2012) asegura:

El aumento en la productividad deriva del uso eficiente de los recursos, por lo que las empresas recurren a varias vías para lograrlo, entre éstas; Fomentar la creatividad e innovación, incrementar la motivación y participación del trabajador, formar y capacitar al elemento humano en el uso de la tecnología para facilitar el trabajo, realizar menos esfuerzo y producir más para mejorar los métodos de trabajo y hacerlos más eficientes, la vida emocional de una mujer violentada se encuentra quebrantada y por ende la motivación que tiene cada una de ellas va a ser mínima, teniendo en cuenta que la motivación es un factor relevante las empresas deben tener un plan de acción para contrarrestar situaciones de maltrato en sus empleados, pues de ellos depende que se alcance el ideal de rendimientos (p. 33).

En Colombia, las empresas dedicadas a la producción de materias primas requieren de personal que esté dispuesto a cumplir con las exigencias del mercado. El Departamento Administrativo Nacional de Estadística - Dane (2015) en la estructura de la industria textil de acuerdo con el tamaño de las empresas, se mantiene invariable entre el 2000 y el 2012, más de un $80 \%$ corresponde a empresas pequeñas, un $16 \%$ a las medianas y un $4 \%$ a las más grandes. Comparativamente, entre el 2000 y el 2010 se crearon 290 empresas nuevas representando un crecimiento del $23.79 \%$. Por número de establecimientos y personal ocupado, por grupo industrial y regional, el $52 \%$ de los establecimientos se dedicaron a la confección de prendas de vestir, excepto prendas de piel (grupo 181), un 30\% a la fabricación de tejidos y artículos de punto y ganchillo (grupo 175), un $9 \%$ a la fabricación de otros productos textiles (grupo 179), el de menor número de establecimientos dedicados fue el de preparación e hiladura de fibras textiles con solo el $1 \%$. Las actividades textiles se concentraron en las regiones de Antioquia, Bogotá (Cundinamarca) y Valle del Cauca.

Según datos del Dane (2016), en noviembre el sector textil-confección registró un crecimiento de $4.8 \%$ en producción, $4.3 \%$ en ventas y $0.7 \%$ en generación de empleo. En el caso de los hilos, tejeduría y acabado de productos textiles registró un alza de $1.5 \%$ en producción y $0.9 \%$ en ventas, mientras que las confecciones crecieron $5.9 \%$ y $4.1 \%$, respectivamente en el mencionado mes. El personal ocupado, en la industria textil y manufacturera, es uno de los más representativos, Antioquia y Bogotá son los de mayor personal ocupado Bonilla y Molano (2014) exponen:

Dentro de la dinámica que presentan estos autores se hace notable de la importancia que tiene el crecimiento textil en Colombia y el personal ocupado en la empresa mas no se toma en cuenta el estado emocional de este personal quien el que se encuentra realizando las funcio- 
nes asignadas, este referente deja visto como las empresas colombianas no tienden a dar relevancia a la necesidad que se hace evidente de conocer las problemáticas de sus empleados y su dinámica familiar (p.3).

Los resultados mencionados brindan un contexto positivo frente a los años anteriores, impactando el empleo especialmente de la mano de obra no calificada de las mujeres. Para Campos (2014) es importante que cada proceso en el clúster de la cadena textil, de confección, de diseño y de moda; muestran que la productividad y competitividad del sector es importante para poder enfrentar retos en los mercados nacionales e internacionales.

En el caso de las tres empresas donde se aplicó el grupo focal y el instrumento se encontró un crecimiento de su producción y contratación de personas, aunque estas empresas se encuentran en la categoría de pequeñas a medianas empresas, buscan tener un personal suficiente que cumpla con los compromisos con sus proveedores.

En la escuela de relaciones humanas se expresa que: "comprender la interacción de los procesos psicológicos y sociales con la situación laboral a fin de influir en el desempeño. Las relaciones humanas fueron el primer acercamiento que enfatizó las relaciones laborales informales y la satisfacción del trabajador" (Bateman \& Snell, 2004, p. 44).

De lo anterior, es valioso mencionar el proceso que se explica entre lo social y la influencia en el desempeño laboral, separar al ser humano de los problemas familiares, académicos o cotidianos del día a día es complejo, lo que se puede hacer es asimilar estas dificultades para enfrentar las situaciones acertadamente. En la relación empresa del sector textil-confección y productividad, la relación entre estrato socioeconómico y labor es muy relevante, debido a que las mujeres de estratos bajos son las que se emplean en este tipo de empresas y las que presentan más situaciones de violencia de tipo familiar.

Para el sector textil-confección, la mano de obra no calificada es fundamental para su proceso productivo, aunque la tecnología es importante para los procesos productivos algunos de esos procesos son manuales. Ejemplo de lo anterior es la revisión y terminación del cocido, generar medidas iguales, empaque y revisión aleatoria de algunos de sus productos. Si para estos procesos una trabajadora no está concentrada se puede ver afectada la calidad y entrega del producto al proveedor.

De otro lado, la teoría del talento humano enfocada en definir que "la administración de recursos humanos es la utilización de las personas como recursos para lograr los objetivos organizacionales" (Mondy \& Noe, 2005). Es entonces fundamental identificar que las personas son el medio para alcanzar el fin, lograr el alcance de los objetivos organizacionales hace que el empleado se sienta valorado al momento de aportar a su organización, pero que, desde la productividad y desempeño, también logra impactar de forma positiva a toda la organización.

Para considerar otro exponente de la teoría de las relaciones humanas, según Guerrero, Pulido y Celis (2019) la jerarquía en la organización debe ir acompañada de autoridad, pero una autoridad que sea respetuosa, el gerente y su equipo de trabajo deben de conservar un conducto formal y respetuoso, así como canales de comunicación determinada por la organización.

Así mismo, en el desarrollo de las relaciones humanas, cada persona tiene un comportamiento favorable o no según el entorno social, familiar y personal, pero que, al momento de llegar a la empresa, debe ubicarse en un contexto diferente buscando que las situaciones positivas o no, sean asumidas por cada individuo que no afecten sus labores en el trabajo (Moreno \& Pérez, 2018).

Para la empresa, las relaciones interpersonales en el campo laboral son complejas y tienen comportamientos asimétricos, es decir, un individuo reacciona al mismo estimulo de una forma diferente. Por eso, es importante crear una cultura organizacional clara, coherente y alineada a la planeación estratégica de la empresa con el fin que los colaboradores aporte al crecimiento y desarrollo de la organización. El que un empleado tenga un comportamiento diferente a sus pares es un llamado para analizar que sucede y tomar acciones rápidas con el fin de no afectar la productividad (Ramírez-Wong, 2019). 
Para que se logre identificar la relación y los costos que representa la violencia intrafamiliar y productividad, podemos analizar un estudio realizado por Ribero y Sánchez (2004), el cual hace referencia a que la violencia intrafamiliar incrementa la probabilidad que la mujer se quede sin empleo de una manera contundente. Así mismo, las situaciones derivadas de esa violencia interna hacen que se tenga riesgo de incrementar problemas de salud. Igualmente, en los estudios realizados por Ribero y Sánchez (2004), determinan que los costos de esa violencia alcanzan un $4 \%$ del producto interno bruto del país.

En conclusión, encontramos que la relación existente entre productividad y violencia intrafamiliar se relaciona con los fenómenos que se presentan para la trabajadora al momento de sufrir alguna de las situaciones de violencia, como son; verbal, psicológica o física. De esta forma se da una interpretación acertada a la forma en que cada proceso realizado por alguien con una situación de violencia dentro de su familia no logrará el rendimiento esperado por parte de la empresa, especialmente del sector textil-confección.

\section{Método}

La metodología utilizada fue bajo un paradigma interpretativo, con un diseño descriptivo, de corte transversal, qué utilizó como herramienta de recolección de información, un grupo focal apoyado con una entrevista semiestructurada. Esto permitió recoger la información de las participantes y analizar por medio de categorías y sub categorías, determinando así los factores propios de cada participante. Así mismo, se realizó la firma del consentimiento informado, permitiendo a cada una de las participantes su previa autorización para la entrevista.

Por consiguiente, es fundamental que en el proceso de investigación como lo manifiesta Hernández, Fernández y Baptista (2014) se considere que:

El enfoque cualitativo se selecciona cuando se busca comprender la perspectiva de los participantes (individuos o grupos pequeños de personas a los que se investigará) acerca de los fenómenos que los rodea, profundizar en sus experiencias, perspectivas, opiniones y signifi- cados, es decir, la forma en que los participantes perciben su realidad (p. 364).

El diseño de investigación utilizado fue de nivel descriptivo, con un diseño no experimental, ya que no se manipularon variables. Lo anterior debido a que el objetivo de la investigación fue conocer los factores relacionados con la violencia intrafamiliar y el impacto en la productividad. Así, la investigación fue abordada desde un paradigma interpretativo que permitió describir el fenómeno propuesto desarrollado en las empresas del sector textil (Toro \& Parra, 2011).

Con el planteamiento anterior, se pretende identificar como la relación de violencia intrafamiliar afecta la productividad laboral, es decir, determinar la causa y el efecto del fenómeno estudiado, considerando que desde la subjetividad de cada individuo se presentan diferentes perspectivas frente al tema.

De otro lado, se utilizó como herramienta de recolección de información una entrevista semiestructurada con apoyo de un grupo focal, según Álvarez-Gayou (2003):

El grupo focal se ha vuelto una de las técnicas favoritas en las investigaciones cualitativas. Si bien es cierto se requieren habilidades en su manejo, las cuales vuelven necesario un entrenamiento previo, constituye una técnica relativamente fácil y por demás interesante, dada la riqueza de información que con ella se obtiene. El grupo focal surge a finales de los años treinta. En esa época, los científicos sociales se planteaban algunas dudas sobre la precisión en la recolección de datos, en cuanto a las partes inherentes a los modos de investigar. (pp. 128-129)

El desarrollo del grupo focal permitió a los investigadores al momento de aplicar el instrumento de identificar otros aspectos no propios de la investigación, pero que tienen alguna incidencia, tales como: el ingreso económico, los gastos, las personas a cargo y el estrato socioeconómico.

La unidad de análisis determinada para esta investigación fue considerada desde la siguiente forma, en las ciudades de Bogotá, Medellín, y Manizales, 24 
mujeres, 8 por cada ciudad, en tres empresas del sector textil confección, lo cual permitió un análisis comparativo, lo que derivó en un proceso de análisis del fenómeno propuesto en cada ciudad, identificando los factores comunes que se presentan de la violencia intrafamiliar.

En concordancia con lo que se expone, el inicio de la investigación estuvo enfocado en establecer los tres grupos de trabajo en cada una de las ciudades, que fueron seleccionados de acuerdo con los intereses de la investigación y según las características de la investigación cualitativa. Con esto se inició ubicación de la unidad de análisis, tarea que desde el inicio presentó dificultad. Algunas de esas situaciones que se presentaron fueron las restricciones laborales que imponían las empresas en las ciudades de Bogotá y Medellín, esas condiciones estaban definidas por falta de tiempo de los gerentes para atender la solicitud de realizar la actividad, la falta de tiempo y los compromisos que se tienen para la entrega de los pedidos lo que puede afectar el trabajo y la disminución de la productividad laboral. Así mismo, se percibió que el clima organizacional en este tipo de empresas es deficiente y existe rivalidad y competencia entre las diferentes áreas, hasta convertirse en una competencia y con relaciones difíciles para el desempeño de las actividades.

Se consideró importante realizar el análisis de las categorías y sub categorías mostradas en la tabla 1. Se agruparon de forma interpretativa las respuestas brindadas y con el apoyo de literatura sobre el tema se presentó la siguiente estructura.

Tabla 2.

Indicadores de violencia en la empresa
Tabla 1.

Categoría y subcategoría del estudio

\begin{tabular}{ll}
\hline \multicolumn{1}{c}{ Categoría } & \multicolumn{1}{c}{ Sub-categoría } \\
\hline Tipología de la violencia & $\begin{array}{l}\text { Física, verbal o psicoló- } \\
\text { gica. }\end{array}$ \\
\hline Infractor de la violencia & $\begin{array}{l}\text { Esposo, novio, compañero } \\
\text { permanente, amigo. }\end{array}$ \\
\hline $\begin{array}{l}\text { Estado emocional post } \\
\text { violencia }\end{array}$ & $\begin{array}{l}\text { Miedo, tristeza, ansiedad, } \\
\text { rabia }\end{array}$ \\
\hline Apoyo gerencial y & $\begin{array}{l}\text { Acompañamiento geren- } \\
\text { cial, psicológico, legal. }\end{array}$ \\
laboral & $\begin{array}{l}\text { Desmotivación, descon- } \\
\text { centración, baja producti- } \\
\text { vidad, ideas de renuncia. }\end{array}$ \\
\hline Desempeño laboral &
\end{tabular}

Fuente: elaboración propia.

De las categorías anteriores, en la tabla 2 se presentan algunos indicadores que las empresas pueden tomar como valoraciones para determinar los efectos de los tipos de violencia y poder medir en el contexto el vínculo que existe entre violencia y la productividad laboral. Esto puede permitir a las empresas tomar acciones preventivas y de mejora cuando este tipo de situaciones sucedan.

La finalidad de los indicadores fue no solo medir la producción, sino acercarse a las realidades que se pueden presentar frente al fenómeno de la violencia intrafamiliar. Este fenómeno se describe en las categorías propuestas para conocer la tipología de la violencia, el generador de la violencia, el estado emocional luego de sufrir situaciones de violencia

\begin{tabular}{lcll}
\hline Variable & Abreviatura & Indicador & Forma de medición \\
\hline Tipología de la violencia & TV & $\begin{array}{l}\text { Tipo de violencia / Total mujeres } \\
\text { violentadas }\end{array}$ & Tipo de violencia \\
\hline Infractor de la violencia & IV & $\begin{array}{l}\text { Generador de violencia / Total } \\
\text { mujeres afectadas }\end{array}$ & Generador de violencia \\
\hline $\begin{array}{l}\text { Estado emocional post } \\
\text { violencia }\end{array}$ & EMV & $\begin{array}{l}\text { Estado emocional / Total mujeres } \\
\text { afectadas }\end{array}$ & Situación emocional \\
\hline $\begin{array}{l}\text { Apoyo gerencial y } \\
\text { laboral }\end{array}$ & AG & $\begin{array}{l}\text { Casos conocidos por los gerentes / } \\
\text { Total casos por mes }\end{array}$ & Caso con apoyo gerencial \\
\hline $\begin{array}{l}\text { Desempeño laboral } \\
\text { Número de unidades día operaria / }\end{array}$ & DL & $\begin{array}{l}\text { Númoductividad } \\
\text { Producción por día }\end{array}$ \\
\hline
\end{tabular}

Fuente: elaboración propia 
intrafamiliar, el apoyo o no de los gerentes de las empresas del sector textil-confección y el desempeño laboral que tiene las colaboradoras al frente de las actividades asignadas.

Las empresas pueden considerar la relación que existe entre una trabajadora que no ha sufrido alguna tipología de violencia, frente a otra que está pasando por esta situación. La diferencia entre los dos escenarios se percibe desde la productividad y la calidad de los productos que se entrega para los respectivos controles que se deben de tener para valorar si una prenda confeccionada cumple con las condiciones exigidas por el cliente.

\section{Resultados}

Los resultados de este estudio muestran que es de vital importancia profundizar en los hallazgos de la investigación. Esto con el fin de reconocer la realidad familiar y relacional de las participantes, por otra parte, entender cuáles se enmarcan en la revictimización en el ámbito laboral, dificultades para el desarrollo de su labor, ocultar su realidad violenta y sometimiento a cargas laborales mayores para no perder su empleo.

Con respecto a las categorías y análisis propuestos, se encontró que los factores emocionales que presentan las mujeres luego de haber sufrido algún tipo de agresión, enmarcada en cualquier tipo de violencia, expresaban puntualmente síntomas asociados a la ansiedad, el miedo, la vergüenza y la zozobra posterior a la agresión. De igual forma, el tener que enfrentarse a sus actividades laborales se constituía en un factor desencadenante en la presentación de síntomas ansiosos. Por otra parte, expresiones de llanto, desolación y sentimientos de desprotección son patrones comunes en las mujeres que participaron de la investigación en las 3 ciudades.

Así mismo, se encontró en las respuestas a la entrevista, que las mujeres presentaban síntomas y evidencias físicas luego de la agresión tales como llanto, nerviosismo, poca concentración, temblor en las manos, acompañados de pensamientos negativos, como por ejemplo: "me van a despedir", "si no cumplo la cuota de producción quizá pierda mi trabajo" "no quiero que mi jefe se entere de lo que me pasó" "va a ser difícil que me den el permiso de ir a hacer la denuncia", entre otros. Estos síntomas se agudizaban al tener que enfrentar el ámbito laboral, teniendo en cuenta la necesidad de solicitar permisos, apoyo psicológico (si la empresa contaba con el servicio), tramitar denuncias (en casos donde se decidía hacerlo) o simplemente evitar perder su empleo.

Ahora bien, toda la sintomatología anteriormente descrita, esta evidentemente asociada con las demandas sociales y familiares que debe cumplir la mujer agredidas. Entre estas están continuar con su labor como madres y de igual forma con su rol de empleadas, lo que tiene unas implicaciones puntuales en la agudización de sus síntomas adversos. Este punto fue recurrente en las tres ciudades, ya que los sentimientos de deseo de abandono de la labor, desgano para la realización de actividades laborales extras, vergüenza por su estado físico (morados en la cara o lugares visibles como brazos como resultado de la agresión) generan un sentimiento de aislamiento total. Es así, como Díaz y Arencibia, (2010) explican que normalmente, la violencia se produce de forma aislada y tiene un comportamiento continuo hasta que la situación es denunciada o evidente entre la familia y personas del trabajo. Ese vínculo de violencia se ha transformado en una situación dolorosa y sinónimo de desigualdad. Lo anterior, se supone como una situación de vulnerabilidad a los derechos humanos y el respeto por el otro. Así mismo, se tiene una transformación constante en la dinámica del poder, de una posibilidad de asumir roles y la manera en que aparecen las diferentes tipologías de violencia.

Haciendo un análisis comparativo en las tres ciudades se encontraron similitudes que se presentan como simétricas y uniformes de acuerdo con el objeto de estudio propuesto en el contexto laboral. en el ámbito laboral. Por ejemplo, dentro de la investigación se evidenciaron rasgos de conductas similares de las mujeres agredidas, en lo que respecta a solicitar ayuda o contar lo sucedido, fue un factor común el no querer expresar lo sucedido, también el factor estresante que era para ellas la posibilidad de perder su empleo y quedarse sin dinero para sostener a su familia. De igual forma en lo que se refiere a permisos de salida, aunque se 
ofrecen a las víctimas para realizar todas las gestiones de denuncia, el tiempo que se ausentan de su labor, deberán reponerlo o tendrán descuentos en el salario.

Por otro parte, en cuanto a la sintomatología asociada al evento traumático, se evidencian estados emocionales comunes en las mujeres de las tres ciudades ya que los síntomas de malestar y desmotivación para asistir a su lugar de trabajo luego de un evento violento se presentaron como un factor común posterior a la agresión.

Otro elemento común encontrado en el desarrollo de la investigación fue la forma de atender estas situaciones en las empresas. En las tres empresas carecían de apoyo a las víctimas, o un plan de apoyo rápido para atender el caso, los permisos ofrecidos en el horario laboral para los trámites de denuncia de violencia intrafamiliar, debieron ser luego repuestos por las víctimas con horas extras de trabajo sin paga, lo que se constituye como factor de estrés adicional a la situación de estas mujeres.

\section{Discusión}

La incidencia en el desempeño laboral luego de haber sufrido agresión física es evidente en esta investigación, las mujeres que participaron activamente en las ciudades de Medellín, Bogotá y Manizales, mostraron coincidencias en cuanto al manejo de sus patrones conductuales, en lo que se refiere a las estrategias de afrontamiento en el ámbito laboral, coincidieron también en las formas de manejo, omisión de la información y miedo a perder su empleo.

La investigación hizo posible mostrar la desatención laboral a la problemática de violencia, ya que en ninguna de las empresas se contempla tener un manejo adecuado de la problemática. Por el contrario, se percibe a la trabajadora como un problema emergente que disminuye la productividad y que puede convertirse en una posible causa para que sea desvinculada de la empresa. Se evidenció desatención, exigencias laborales de cumplimiento en cuanto a las horas prestadas para permisos de denuncias y citas médicas, seguimiento a la víctima como medida de control de posibles ausencias a posteriori y en algunos casos amenazas con el término del contrato laboral.

Según el estudio realizado por Medicina Legal en el año 2015 da cuenta de que la situación de la mujer agredida en Colombia, es crítica, ya que tomando estudios asociados como el realizado por Licona y Otero (2015) que analizaron los factores sobre los efectos de la violencia de género en la productividad laboral de la mujer en la ciudad de Cartagena en el periodo 2004 - 2013, y está investigación describen una realidad desalentadora, lejos de constituirse el ámbito laboral en un espacio de apoyo y propósito de disminución de la problemática, más bien se presenta como factor estresor y hasta acusatorio hacia la mujer víctima del fenómeno asociado.

A partir de lo expuesto, se encontró que como consecuencia, las victimas preferían callar u omitir información acerca de los episodios violentos sufridos antes de contarlo a sus compañeros de trabajo, jefes o superiores, ya que a pesar de necesitar el apoyo, era más intenso el miedo que sentían a perder su trabajo.

Un factor adicional, fue que en ninguna de las empresas se encontró una ruta de atención que ofreciera apoyo a las mujeres víctimas de violencia, incluso, en ninguna de ellas había servicio de Psicología. Así, lo que se hizo fue realizar la remisión a la Entidad Prestadora de Salud (EPS), la Fiscalía y a Medicina Legal, cuando se presentaban marcas en el cuerpo de las víctimas. En caso contrario, situaciones como el asecho, las amenazas y el hostigamiento no representaron un peligro tangible y por lo tanto no se tenían estipuladas acciones en las empresas.

Así mismo, dentro de las empresas el fenómeno de la violencia no es tomado en cuenta como un factor importante para la calidad de vida de sus empleados, por eso, los permisos o solicitudes que las mujeres realicen son argumentados bajo otras excusas. Es decir, permisos de otra índole donde su salario no se vea afectado y menos que se evidencie que su situación personal fue permeada por aspectos de violencia intrafamiliar.

El proyecto de investigación se realizó con el fin de encontrar relaciones de tipo personal, familiar 
y social; y como lo anterior puede generar en las trabajadoras especialmente de este sector a disminuir su productividad, calidad laboral y estabilidad, generando así situaciones de rotación de personal, fallos en los procesos de mejora continua, perdida de la curva de aprendizaje y no lograr conformar un equipo de trabajo estable y sólido.

Se deja abierta la opción para que otro tipo de estudios se enfoquen desde un corte cuantitativo, buscando una muestra más representativa, caracterización de la calidad de vida, nivel socioeconómico, ingresos, núcleo familiar entre otras variables que permitan medir el fenómeno estudiado.

\section{Conclusiones}

La violencia intrafamiliar tiene impacto en la vida personal, social, cultural, laboral y afectiva de cualquier persona, en este caso sobre la mujer. El objetivo de la investigación fue identificar como la violencia intrafamiliar afecta la productividad laboral, se hace necesario, que los estudios anteriores frente a una problemática social de violencia de género contemplen que existen otros factores asociados a la situación de la violencia familiar como lo es no tener un empleo por haber sufrido y perdido el que se tenía.

Los resultados encontrados en la investigación son valiosos, ya que evidencian una problemática social grave. Las estadísticas en crecimiento indican que múltiples factores de riesgo de violencia afectan a las mujeres en su ser, y tiene un impacto directo en el desempeño laboral. En las lógicas de la violencia intrafamiliar, se atraviesan situaciones individuales, familiares, sociales y laborales, que en conjunto, derivan en problemáticas empresariales que aún no tiene visibilidad y trazabilidad por parte de los gerentes.

Igualmente, el estado emocional, como la manifiesta Casique (2014) el hombre tiene un rol en la sociedad, en Colombia el comportamiento machista, resuelve los problemas con violencia y no por el diálogo. Es por eso que las empresas pueden establecer caracterizaciones o estudios internos con el fin de identificar cómo está compuesto el núcleo familiar de sus trabajadores, para una respuesta a situaciones negativas que se puedan presentar con una trabajadora por estar o haber sufrido alguna tipología de violencia.

La violencia intrafamiliar contra la mujer constituye no solo un proceso de aislamiento y sometimiento, sino que se convierte en un camino para acelerar decisiones catastróficas, como puede ser el suicidio (Sánchez, 2013). Lo anterior debería ser conocido por la empresa y las entidades del estado, con el fin de crear rutas de atención para que la violencia intrafamiliar no vaya a generar problemas de salud pública o incrementos en las tasas de suicidios.

Un modelo preventivo frente a la violencia intrafamiliar puede ser diseñado por parte de autoridades públicas y las mismas empresas. Algunas estrategias pueden ser capacitaciones, talleres, cursos cortos, que logren afrontar las situaciones que se desarrollan en el grupo familiar y desde la misma empresa. Al descifrar la problemática se debe conocer con claridad cómo serán atendidos los casos de violencia.

Así las cosas, podemos concluir que la violencia intrafamiliar no se elimina, sino existe una cultura de cambio y transformación en la sociedad. La violencia contra el género femenino es una realidad, se debe crear estrategias que cambien estos comportamientos que afectan a la base de la sociedad, la familia y que tiene un impacto directo en la productividad de las empresas. Desde las organizaciones, crear planes de atención y realizar seguimiento, permitirá mejorar la calidad de vida y que la productividad y competitividad de la empresa no se vea afectada por este tipo de problemas sociales.

\section{Referencias}

Álvarez- Gayou, J. (2003). Cómo hacer investigación Cualitativa. Fundamentos y Metodología. México: Paidós.

Álvarez, A. \& Gómez, I. (2011). Conflicto trabajo - familia, en mujeres profesionales que trabajan en la modalidad de empleo. Revista pensamiento psicológico, 9(16), 89-106. 
Bateman, T. \& Snell, S. (2009). Administración. Liderazgo y colaboración en un mundo competitivo. México: McGrawHill.

Bonilla O. E. \& Molano Aponte, L. (2014). La dinámica de la productividad en la industria textil de Colombia 2000-2010. Observatorio de la Economía Latinoamericana, No 199, 2014. Recuperado de http://www.eumed.net/cursecon/ecolat/co/14/industria-textil.html

Campos, A. G. (2014). El sector textil en Colombia, ¿cómo ser más competitivos?. (Trabajo de especialización en gerencia en Comercio Internacional). Universidad Militar Nueva Granada, Bogotá. Colombia.

Casique, I. (2014). Empoderamiento de las jóvenes mexicanas y prevención de la violencia en el noviazgo. Revista papeles de población, 20(82), 27-56.

Castillo, M. (2011). Violencia de pareja en el Paraguay según la Encuesta Nacional de Demografía y Salud Sexual y Reproductiva 2008, Revista Latinoamericana de población, 5(9), 27-48.

Castro, R. \& Casique, I. (2008). Violencia de género en las parejas mexicanas, resultados de la Encuesta nacional sobre la dinámica de las relaciones en los hogares 2006. México: Instituto Nacional de las mujeres - Inmujeres. Recuperado de http://cedoc.inmujeres.gob. $\mathrm{mx} /$ documentos_download/100925.pdf

Cruelles, J. (2012). Productividad e Incentivos. Cómo hacer que los tiempos de productividad se cumplan. Barcelona, España: Editorial Marcombo.

Departamento Administrativo Nacional de Estadística - DANE. (2015). Encuesta anual del comercio 2014 (Boletín de Prensa EAC2014). Recuperado de https://www.dane.gov. co/files/investigaciones/boletines/eac/bol_ eac_2014_def.pdf

Departamento Administrativo Nacional de Estadística - DANE (2016). Encuesta anual del comercio 2014 (Boletín de Prensa EAC-
2014). Recuperado de https://www.dane.gov. co/files/investigaciones/boletines/eac/bol_ eac_2016.pdf

Díaz, R. \& Arencibia, F. (2010). Comportamiento de la violencia intrafamiliar en asistentes a consulta de Psicología. Revista Médica Electrónica, 32(2), 34-43.

Fuentes, S. (2012). Satisfacción laboral y su influencia en la productividad. (Tesis de pregrado). Universidad Rafael Landivar. Quetzaltenango. México. Recuperado de http://biblio3.url.edu. gt/Tesis/2012/05/43/Fuentes-Silvia.pdf

García Figueroa, G. (2014). Violencia intrafamiliar y divorcio: las contradicciones entre los dichos legales y los hechos conservadores en Hermosillo, Sonora. Revista región y sociedad, 4, 217-260.

González, V. (2018). La violencia laboral desde una perspectiva de género en la Administración Pública de la provincia de Corrientes. Revista Pilquen, 21(4), 10-21.

Guerrero, M., Pulido, A., \& Celis, Y. (2019). Impacto de las relaciones humanas en la competitividad de las asociaciones. Revista UIS Ingenierías, 18(1), 61-72. DOI: 10.18273/revuin. v18n1-2019005

Hernández, R. C, Fernández, F. C. \& Baptista, P. (2014). Metodología de la investigación. México: McGraw Hill.

Hidalgo, L., \& Valdés, D. (2014). Violencia contra la mujer adulta en las relaciones de pareja. Revista Medisan, 18(2), 177-182.

Instituto de Medicina legal en el informe Forense. (2015). Boletin Epidemiológico Información Estadistíca de Violencia contra la Mujer. Recuperado de http://www.medicinalegal. gov.co/documents/20143/49523/Violencia+int rafamiliar+primera+parte.pdf

Licona, G \& Otero, J. (2015). Efectos de la violencia de género en la productividad laboral de la mujer en la ciudad de Cartagena 
en el periodo 2004-2013. (Trabajo de grado de pregrado). Universidad de Cartagena, Cartagena. Colombia.

Mondy, R. \& Noe, R. (2005). Administración de Recursos Humanos. México: Pearson Education.

Moreno, S. \& Pérez, E. (2018). Relaciones interpersonales en el clima laboral de la universidad tecnológica del Chocó Diego Luis Córdoba, Revista Ces, 9(1), 13-33.

Pacheco, A. \& Capano, A. (2014). Estrés y violencia domestica: un estudio en adultos referentes de niños, niñas y adolescentes. Revista de la Universidad Católica de Uruguay, 7(1), 31-42.

Quiñones, M., Arias, Y., Delgado, E., \& Tejera, H. (2011). Violencia intrafamiliar desde un enfoque de género. Revista Medigraphic, 17(2), 1-10.
Ramírez-Wong, L. (2019). Relaciones interpersonales en el ámbito laboral. (Tesis de maestría). Universidad Instituto Tecnológico y de Estudios Superiores de Occidente. Jalisco, México.

Ribero, R., \& Sánchez, F. (2004). Determinantes, efectos y costos de la violencia intrafamiliar en Colombia. Documento CEDE - Universidad de los Andes. https://economia.uniandes.edu. co/components/com_booklibrary/ebooks/ D2004-44.pdf

Sánchez, G. (2013). ¡Basta ya! Colombia: memorias de guerra y dignidad. Recuperado de http:// www.centrodememoriahistorica.gov.co/descargas/informes2013/bastaYa/resumen-ejecutivo-basta-ya.pdf

Toro, J., \& Parra, R. (2011). Fundamentos epistemológicos de la investigación y la metodología de la investigación. Medellín, Colombia: Editorial Eafit. 